\title{
Article \\ Quantifying Differences among Ten Fingers in Force Control Capabilities by a Modified Meyer Model
}

\author{
Cong Peng $1 \oplus$, Dangxiao Wang ${ }^{1,2,3, *}$ and Yuru Zhang ${ }^{1,2} \oplus$ \\ 1 State Key Laboratory of Virtual Reality Technology and Systems, Beihang University, Beijing 100083, China \\ 2 Beijing Advanced Innovation Center for Biomedical Engineering, Beihang University, Beijing 100083, China \\ 3 Peng Cheng Laboratory, Shenzhen 518055, China \\ * Correspondence: hapticwang@buaa.edu.cn; Tel.: +86-010-823-380-23
}

Received: 16 July 2019; Accepted: 28 August 2019; Published: 3 September 2019

\begin{abstract}
Quantifiable differences among fingers in force control capability have both important practical and theoretical values in characterizing force control of accurate finger-tapping tasks. Following the classical Fitts' law paradigm, we quantified the differences among ten fingers in term of speed-accuracy trade-off (SAT) in performing repetitive discrete force control tasks. Visual cues displaying targeted force magnitudes and tolerances were provided. Users were required to apply the targeted force within the given tolerance quickly and accurately by pressing a force sensor using the specified finger. We found that ten fingers obeyed the Meyer model in the SAT performance and they differed in reaction time, the index of performance (IP), and the goodness of fit for the Meyer model. A modified Meyer model was proposed to quantify the difference between ten fingers in the SAT performance using only one parameter, making the quantification easier than using the original Meyer model. Pairwise comparisons showed that the differences between symmetrical fingers on both hands were insignificant except for the pair of index fingers. These findings provided us with multiple perspectives on the differentiation among ten fingers in the force control capabilities. Our study helps lay the foundation for engineering systems that rely on finger force control ability.
\end{abstract}

Keywords: behavioral biometrics; force control capability; finger difference; Fitts' law; speed-accuracy trade-off

\section{Introduction}

Recently, practical and theoretical values of exploring the differences in executive capability among fingers have been emerging. Many of our daily activities and especially dexterous manipulative actions rely heavily on the remarkable versatility of different fingers on our hands [1]. A lot of research has shown that individual fingers differ in their facility of usage [2-4], and different fingers are crucially involved in an extensive repertoire of daily manipulative tasks, such as piano playing [5] and keystrokes in typing [6,7]. For example, a common requirement for multi-finger interactive devices is to assign a different key to each of the fingers [8-10]. Consequently, exploiting the ability differentiation between fingers to apply force in interactive designs [11,12] are becoming necessary, because quantifiable differences among the ten fingers may provide a basic understanding for an interactive method to facilitate individualized designs $[13,14]$, e.g., a haptic keyboard [15,16]. Furthermore, a pending issue that needs to be addressed is whether we should choose either the same-hand index finger and middle finger or the different-hand symmetrical fingers for the best pair of responding fingers in force control tasks to minimize the influence of individual finger differences on behavioral measures $[17,18]$.

The investigation of the effects of the individual finger on total force production is one of the research hotspots in the field of multi-finger synergy. Latash et al. [19-22] studied the control of multi-finger force synergy based on maximal voluntary contraction (MVC) force tasks and proposed 
an uncontrolled manifold analysis for multi-finger synergy. They found that when individuals produced either maximal or sub-maximal force levels with one finger on the right hand, the other fingers involuntarily contributed to the total force production. Their results indicated that the index finger showed the lowest enslaving while the ring finger showed the largest enslaving indices. Kinoshita et al. [23] examined the contributions and co-ordination of external finger grip forces during a lifting task with a precision grip using multiple fingers. They revealed that the percentage contribution of the static grip force to the total grip force for the index, middle, ring, and little fingers was $42.0 \%$, $27.4 \%, 17.6 \%$, and $12.9 \%$, respectively. Similarly, Kong et al. [24] studied the difference of total grip strength and individual finger force between dominant and non-dominant hands in various grip spans of pliers. Kong et al. found that the difference of the individual finger force on the total grip strength between the dominant and non-dominant hands was not the same as the difference of total grip strength.

In terms of single-finger tasks, performances among different fingers were also quantified by reaction or force control. Gatewood [5] investigated the speed and accuracy of the reactions of ten fingers in a keystroke in reaction to visual stimuli. In his experiment, the performances of individual fingers were measured with a percentage of correct response for a given time limit. The response speed was indicated by the time limit, and the response accuracy was indicated by the correct percentage. He revealed measurable differences among the reactions of individual fingers in speed and accuracy, respectively. In terms of the performance of fast, repetitive tapping movements, Aoki et al. [2] demonstrated different results for four fingers of the right hand in maximum tapping cadence, movement velocity, and key-contact forces generated from each of the four different fingers. The index finger attained the highest values in these three indexes, while the values were progressively lower for the middle, little, and ring fingers, respectively. Nevertheless, differences in the speed-accuracy trade-off (SAT) of individual fingers were not taken into account. The SAT is a classical behavioral metric widely used to quantify motion control performances between different users or tasks [25]. The SAT offers a useful framework to study movement processes, because it reflects the everyday compromise in human behavior, as conflicting constraints of speed and spatial accuracy require constant modulation of voluntary movement [26].

Increasing studies are devoted to extending Fitts' law to finger force control tasks [27,28], as this classical law has been confirmed over a variety of motion control tasks $[29,30]$, and frequently used to quantify user performance and motor control system function [31,32]. Fitts made one of the most iconic studies in modeling the SAT of human motor behavior [33]. Classical Fitts' law states that the movement time (MT) of an aiming movement was a linear function of the index of difficulty $[29,30]$. The index of difficulty (ID) is defined as the amount of information necessary to select a specific amplitude from the total range of possible movements [25]. Accordingly, Gorniak et al. [27] explored the possible effects of a negative co-variation among finger forces in multi-finger accurate force production tasks on the classical Fitts' speed-accuracy trade-off. In their experimental tasks, subjects performed cyclic force changes between pairs of targets "as quickly and accurately as possible". Gorniak et al. found that there was a close-to-linear relationship between the standard deviation of force amplitude and movement time (MT). No differences between the performance of either of the two "radial" fingers (index and middle) were observed. The "ulnar" fingers (little and ring) showed higher indices of variability and longer movement times as compared to both "radial" fingers. However, the performance of fingers was not compared by the index of performance (IP) defined in Fitts' law tasks.

As the reciprocal of the slope in a unit of "bits/s", the IP tends to be a constant to characterize and compare users' performance with different devices and in different movement contexts [30]. The IP has been interpreted as throughput indexing the information processing capacity for executing a given class of movements from the perspective of information theory [34,35]. Gorniak et al. [27] openly admitted that conclusions drawn from their study might be task-specific and could not be generalized to discrete force production tasks. In discrete and repetitive force control tasks with discrete visual/audio/haptic feedback cues, Li et al. [28] confirmed that the performance of the index finger was also in accord 
with the Meyer model that was well-known as an extension of the definition of task difficulty in Fitts' law [29]. In one of our previous work, the IP of the Meyer model was employed to quantify the differences among the fingers of the right hand [36]. In summary, the tasks and methods for quantifying the performances among fingers have been evolving over the past years. The differences between all ten fingers have not been fully revealed and quantified in repetitive discrete force control tasks.

In this paper, we investigated whether all ten fingers are different in the performance of the speed-accuracy trade-off (SAT) in repetitive discrete force control tasks. Firstly, we measured reaction times in the discrete force control tasks with different levels of difficulty. Then the relationship between reaction times and difficulty levels was checked for each finger by regression analysis of Meyer model. Secondly, the differences between all ten fingers were quantified by reaction time, $I P$, and goodness of fit $\left(R^{2}\right)$. A modified Meyer model was proposed for pairwise comparisons between individual fingers.

The main contributions of this paper include the following:

- The differences among all ten fingers in force control capabilities were revealed and characterized quantitatively.

- A modified Meyer model was proposed for quantifying and comparing the difference between ten fingers in the performance of SAT in the discrete force control tasks.

\section{Materials and Methods}

\subsection{Subjects}

The subjects in this study were 12 healthy young students (ages 22-29 years, $M=25.3, S D=1.9$, four females) from Beihang University. They all reported normal or corrected-to-normal vision acuity and were right-handed according to their preferential use of the hand during daily activities. None of the participants had a previous history of neuropathies, traumas to the upper limbs, or long-term involvement in hand or finger activities such as typing or playing musical instruments. The research was conducted following the Declaration of Helsinki. All participants provided their written informed consent before the experiment and were informed of their rights to discontinue their participation at any time. Each of them received a bonus of $¥ 50$ (about \$15) after the test was finished.

\subsection{Apparatus}

The experimental apparatuses have been improved based on the system used in our previous study [18]. In the previous measurement system as shown in Figure 1, a virtual isolated testing environment for the stimulus-reaction task was developed using the commercial software Unity 3D (Unity Technologies, Brighton, UK). The virtual environment was presented by a head-mounted display (Oculus Rift CV1, Oculus Inc., Saint Louis, MO, USA) for experiments of finger force control tasks. A time-minimization movement task with a radial spatial constraint was adopted [37]. There were four semi-transparent cylinders in the virtual environment. At the bottom of each cylinder, there was a color disk that was able to move up and down in the cylinder. The height of a color disk increased along with the magnitude of the actual key-pressing force $\left(F_{R}\right)$, which was measured by force sensors (FSG15N1A, Honeywell International Inc., Morris Plains, NJ, USA; diameter, $5 \mathrm{~mm}$; minimum resolution, $0.01 \mathrm{~N}$; response time, $1 \mathrm{~ms}$; measurement range, 0-15 N) embedded in a customized rectangular box. Amplified force sensor signals from the finger force measuring system were digitized via a 12-bit A/D converter at a sampling rate of $200 \mathrm{~Hz}$ for each channel and then stored in a data acquisition module (AD MP4623, WWLAB, Beijing, China). Each sensor was covered with a rubber cap to increase friction and prevent the influence of finger skin temperature on the force measurements. The signal from each sensor was set to zero at the beginning of the experiments. A visual cue (the grey thick slice) was defined by the force parameters. 

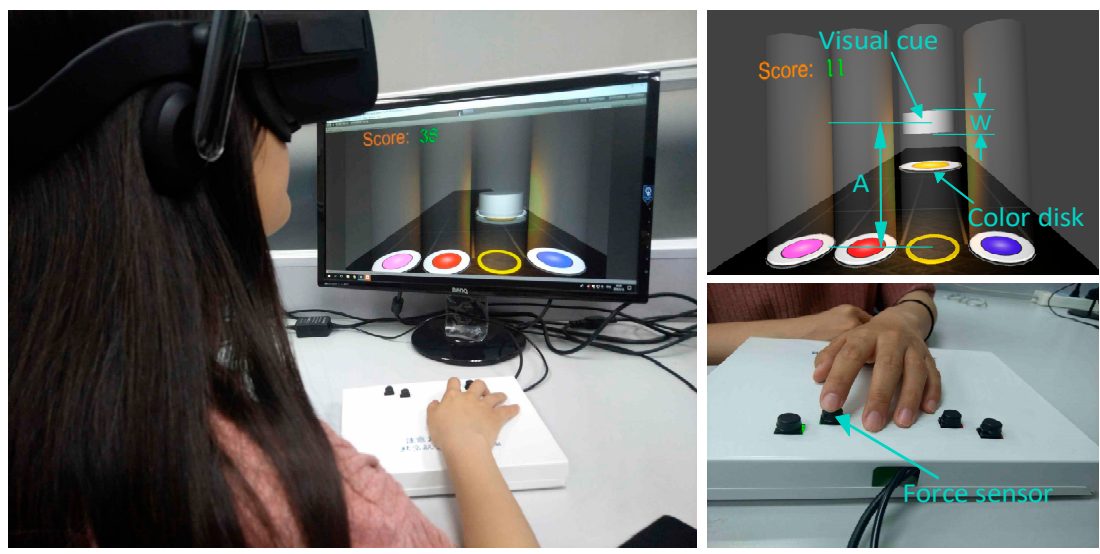

Figure 1. Components of the prototype and the experimental scenario.

Unlike the multi-finger pressing in our previous studies, our interest in this paper was single-finger pressing, hereby we only activated the yellow disk in the cylinder labeled with "RI", and deactivated other disks in the experiment. A set of force control measurements for single-finger tapping were reprogrammed and conducted in the virtual environment shielding external audiovisual interference.

\subsection{Single-Finger Force Control Tasks}

A fast-paced stimulus-reaction force control task was adopted leveraging Fitts' reciprocal tapping task. In accord with the paradigm of the distance-constrained tasks [37], we used a fixed set of ID values in our discrete finger force control tasks. As shown in Figure 1, the height and thickness of the cue indicated the magnitude (A) and the tolerance (W) of the target force. There were 15 pairs of $A$ and $W$. $A$ was selected from the set $(1,2$, and $3 \mathrm{~N})$, while $W$ was selected from the set $(0.2,0.3$, $0.5,0.7$, and $0.8 \mathrm{~N})$. The tolerance range was defined symmetrically with respect to the magnitude as: $(\mathrm{A} \pm 0.1, \mathrm{~A} \pm 0.15, \mathrm{~A} \pm 0.25, \mathrm{~A} \pm 0.35$, and $\mathrm{A} \pm 0.4)$. Therefore, a total of 15 pairs $(3 \times 5)$ of $\mathrm{A}$ and $\mathrm{W}$ combinations were generated. When a visual cue pops up, the subject must quickly move the color disk into the visual cue and keep it inside. Figure 2 showed the continuous variation of $F_{R}$ and the corresponding relative positions between the color disk and the visual cue. For example, when $F_{R}$ was within the target tolerance $W$, the color disk overlapped with the grey thick slice.

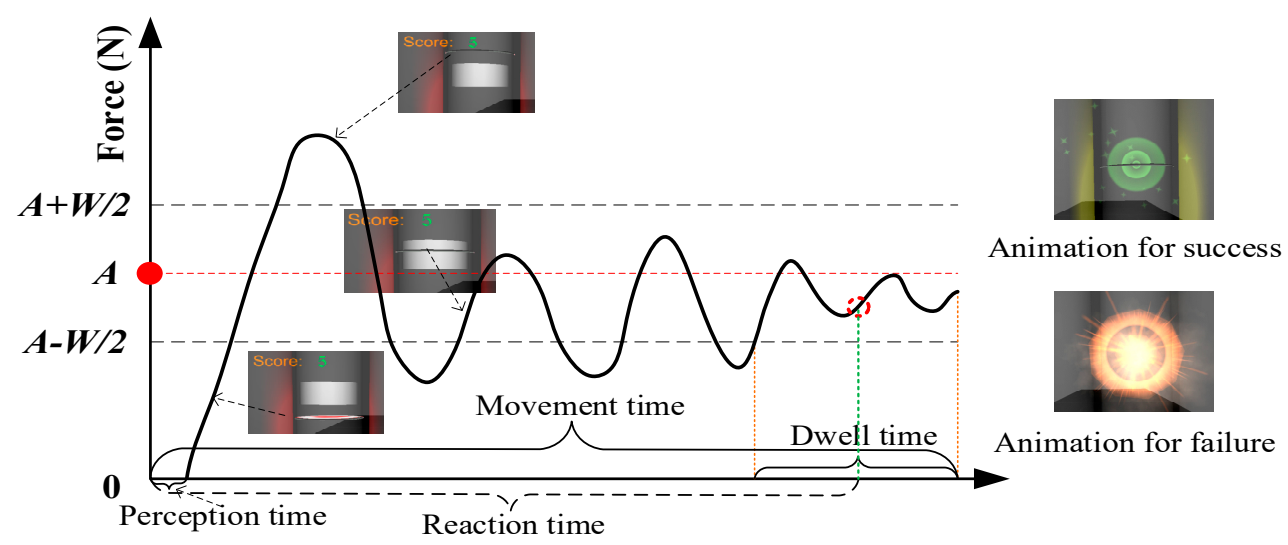

Figure 2. Three states of an example profile of the actual force with respect to the required force range. The position of the circle marked by dotted red lines in the epoch of the dwell time varies with force trials.

In each trial, the subject was required to increase the key-pressing force to meet a given $A$ and $\mathrm{W}$ combination (a target force with a tolerance) as quickly as possible, and then keep the force for a "dwell time" $\left(T_{\mathrm{D}}\right)$. The $T_{\mathrm{D}}$ for maintaining the color disk in the visual cue should be greater than a 
predefined threshold time of $200 \mathrm{~ms}$. In order to complete a force control trial, if $F_{\mathrm{R}}$ jumped out of the $\mathrm{A}$ and $\mathrm{W}$ combination, as shown in Figure 2, the subject needed to adjust his/her key-pressing force to enter the target region again until the dwell time was over. A random sequence of the $15 \mathrm{~A}$ and $\mathrm{W}$ combinations was assigned to a finger in a session. A restriction was designed for the session, that is, each combination appeared only once and was consecutively repeated five times (trials). Therefore, one session consisted of 75 trials. In a session for each of ten fingers, all $\mathrm{A}$ and $\mathrm{W}$ combinations were tested in the pseudorandom order described above. The ten fingers were labeled with LT, LI, LM, LR, LL, RT, RI, RM, RR, and RL wherein the first capital denoted the hand to which the fingers belong ( $\mathrm{L}=$ left, $\mathrm{R}=$ right $)$, and the second capital denoted abbreviation of fingers, $\mathrm{T}=$ thumb, $\mathrm{I}=$ index finger, $\mathrm{M}=$ middle finger, $\mathrm{R}=$ ring finger, and $\mathrm{L}=$ little finger. After the completion of a trial, the responding finger must be lifted completely to prepare for the next trial. For an upcoming trial with a new $\mathrm{A}$ and $\mathrm{W}$ combination, no explicit hints were provided for the changed target force and target tolerance, and subjects needed to engage top-down attention to the reaction using finger force control. It is noted that movement time (as shown in Figure 2) was defined as the time taken from the appearance of a visual cue to the completion of force control during one trial. Terminating at the circle marked by dotted red lines, the actual reaction time $\left(T_{R}\right)$ was calculated by subtracting a half of the $T_{D}$ (100 ms) from the movement time in each force trial. Because a fixed set of $\mathrm{A}$ and $\mathrm{W}$ combinations were used in the current tasks, each reaction would be terminated at the lower or upper bounds of target tolerance once excluding the $T_{\mathrm{D}}$ completely. Whereby, an observed movement might undershoot the target on some trials, while an overshoot might occur on the other trials [29]. If $T_{R}$ was measured in these movements, it would be very inconsistent with the classic Fitts' reciprocal tapping paradigm [33]. Therefore, half of $T_{\mathrm{D}}$ was taken into account for each reaction to reach any location in the target range.

As a component of the human visual and cognitive process, the perception time (as shown in Figure 2) at the beginning of each trial was independent of movement task parameters $[34,35]$. As a result, two measures were taken to avoid the larger perception time caused by distraction as much as possible. First, based on the results of a pilot study, we confirmed that $5 \mathrm{~s}$ was enough for all healthy subjects to complete all the trials. Therefore, the allowable reaction time $5 \mathrm{~s}$ was a time duration in which subjects must finish each force control trial. The trial would be successful if subjects completed the force control reaction within the allowable reaction time, otherwise, it would be a failure. Second, we provided subjects with immediate audio-visual feedback at the end of each trial to engage them during measurements. Animation of successful or failed feedback for each trial followed the disappearance of the visual cue, hereby reminding subjects of the end of the current trial and the beginning of the next one. In addition, the subject would get a score once he or she was successful in a trial. It was hypothesized that encouraging subjects to achieve as many scores as possible would motivate them to maintain continuous vigilance for incoming cues combined with a readiness to react.

\subsection{Procedures}

The procedure of the experiment was explained to the subjects by a text description before experiments began. Before starting a task, the subject was required to wear the earphone and the head-mounted displayer (HMD), and ensure a clear view by adjusting the HMD appropriately. During the whole experiment, subjects sat in a height-adjustable office chair as shown in Figure 1. All subjects were instructed to find a comfortable sitting position that was conducive to normal execution of finger force according to their daily preferences in office. Every subject was required to maintain the upper arm posture and only change the forearm posture when switching the responding finger during the tests. To this end, the position of the force sensor box was adjusted to minimize the changes in positions of his or her forearm and hand. Subjects were asked to move one finger in every trial by bending and stretching the metacarpophalangeal (MP) joint, and repeatedly press the center of the force sensor with the tip of the specified finger. The other nontapping fingers on both hands were required to keep a comfortable and consistent gesture to minimize their effects upon the compression force exerted by the responding finger during the tapping tasks. Subjects were instructed to make a fist 
with the nontapping fingers with the thumb pressing the force sensor vertically, in order to keep the gesture of each nontapping finger consistent with others to the greatest extent possible.

Before starting the formal experiment, every subject had an opportunity to practice freely until he/she judged that he/she could be capable of all combinations across all fingers. The formal experiment was divided into four blocks on four consecutive days. A new random order of the ten fingers was generated for every subject in each block. The sequences for all of the combinations were also generated randomly for each finger in a session. There were rest intervals for $2 \mathrm{~min}$ between consecutive sessions to reduce the influence of fatigue. During the rest period, subjects were asked to provide feedback about their feelings and emotional states by self-evaluation. If subjects claimed they were fatigued between scheduled rest intervals, an additional rest period of 3-5 min was given to the subject immediately. Moreover, if a subject reported that he or she failed to perform the tapping or complete a trial as we instructed in the text description, the subject would be considered to be distracted or tired in the session. The session was required to be repeated once again after an additional rest period. All subjects were able to perform all trials without obvious difficulty.

\subsection{Data Analysis and Statistics}

From a practical point of view, moderate concentration is common in the daily dynamic interactive response. The trial repetition, related learning effect, and inattention, to some extent, contributed to some diversity in the observed outcomes of different fingers [17,18]. Thus, the quickest reactions resulting from automatic behavior and the slowest reactions resulting from distraction were discarded [38]. As was reported by Gorniak et al. [27], some subjects needed practice trials for A and W combinations with high ID values (ID $=3.5$ and 4.0 ). On average, subjects performed one practice session before all IDs 3.5 and 4.0. After visual inspection of the raw data from our measurements, generally constant and short reaction times were observed from the 2 nd trial to the 5 th trial after the onset of each combination in a session. Thus, restricting data analysis by removing the first trial per testing combination was considered to be reasonable in each session. Similarly, in this paper, 192 trials (4 repetitions, 4 sessions, and 12 subjects) were carried out for each finger in every testing combination. Thereafter, the mean of $T_{\mathrm{R}}$ in repetitive trials was calculated for each finger, respectively, after removing the trials with the longest and shortest $T_{\mathrm{R}}$ among all trials of one finger under the same combinations.

The data are presented in the text and figures as means and standard errors. Mean values and standard deviations (in brackets) of $T_{R}$ for all subjects were centrally presented in a table across 15 combinations for ten fingers, as shown in Table 1. SPSS software version 21.0 (SPSS Inc., Chicago, IL, USA) was used for statistical significance test and a $p$-value of less than 0.05 was considered statistically significant. A two-way analyses of variance (ANOVAs) were performed on the average reaction times with the factors: subjects, fingers, hands (left and right hands), and A and $\mathrm{W}$ combinations. These factors were selected depending on particular comparisons. Pair-wise comparisons were performed using Bonferroni statistics to analyze significant effects.

Matlab R2016b (Mathworks Inc., Natick, MA, USA) was used off-line for regression analysis in accordance with Fitts' law. Although the logarithmic model proposed by Shannon based on Fitts' law is most frequently used in human-computer interaction, the Meyer model has been validated to be more suitable for the accurate fingertip force control task $[29,36]$. In this original Meyer model, the reaction time $T_{\mathrm{R}}$ can be quantifiable by the model described in Equation (1), where $\boldsymbol{a}$ and $\boldsymbol{b}$ are regression coefficients characterizing an input system, and ID refers to the index of difficulty in a given $\mathrm{A}$ and $\mathrm{W}$ combination.

$$
\left\{\begin{array}{l}
T_{\mathrm{R}}=a+b \cdot I D \\
I D=\sqrt{A / W}
\end{array}\right.
$$


Table 1. Summary of reaction time $\left(T_{\mathrm{R}}\right)$ across all subjects with respective mean values and standard deviations (in brackets) for ten fingers and $15 \mathrm{~A}$ and $\mathrm{W}$ combinations.

\begin{tabular}{|c|c|c|c|c|c|c|c|c|c|c|c|}
\hline$A$ and $W$ & ID & LL & LR & LM & LI & LT & RT & RI & $\mathbf{R M}$ & RR & RL \\
\hline $1 \& 0.2$ & 2.236 & $\begin{array}{c}0.610 \\
(0.318)\end{array}$ & $\begin{array}{c}0.592 \\
(0.279)\end{array}$ & $\begin{array}{c}0.540 \\
(0.257)\end{array}$ & $\begin{array}{c}0.574 \\
(0.285)\end{array}$ & & & & & & $\begin{array}{c}0.686 \\
(0.332)\end{array}$ \\
\hline $1 \& 0.3$ & 1.826 & $\begin{array}{c}0.520 \\
(0.249)\end{array}$ & $\begin{array}{c}0.474 \\
(0.247)\end{array}$ & $\begin{array}{c}0.467 \\
(0.254)\end{array}$ & $\begin{array}{c}0.505 \\
(0.272)\end{array}$ & $\begin{array}{c}0.530 \\
(0.305)\end{array}$ & $\begin{array}{c}0.513 \\
(0.265)\end{array}$ & $\begin{array}{c}0.438 \\
(0.229)\end{array}$ & $\begin{array}{c}0.455 \\
(0.250)\end{array}$ & $\begin{array}{c}0.451 \\
(0.238)\end{array}$ & $\begin{array}{c}0.521 \\
(0.274)\end{array}$ \\
\hline $1 \& 0.5$ & 1.414 & $\begin{array}{c}0.361 \\
(0.199)\end{array}$ & & & & & & & $\begin{array}{c}0.341 \\
(0.177)\end{array}$ & & $\begin{array}{c}0.385 \\
(0.202)\end{array}$ \\
\hline $1 \& 0.7$ & 1.195 & $\begin{array}{c}0.323 \\
(0.165)\end{array}$ & & & & & & & & & $\begin{array}{c}0.319 \\
(0.171)\end{array}$ \\
\hline $1 \& 0.8$ & 1.118 & $\begin{array}{c}0.294 \\
(0.147)\end{array}$ & & & & & & & & & $\begin{array}{c}0.301 \\
(0.170)\end{array}$ \\
\hline $2 \& 0.2$ & 3.162 & $\begin{array}{c}0.744 \\
(0.338)\end{array}$ & $\begin{array}{c}0.667 \\
(0.309)\end{array}$ & $\begin{array}{c}0.636 \\
(0.277)\end{array}$ & $\begin{array}{c}0.664 \\
(0.292)\end{array}$ & $\begin{array}{c}0.703 \\
(0.321)\end{array}$ & & & & & $\begin{array}{c}0.784 \\
(0.359)\end{array}$ \\
\hline $2 \& 0.3$ & 2.582 & & & & & & & & & & $\begin{array}{c}0.666 \\
(0.348)\end{array}$ \\
\hline $2 \& 0.5$ & 2.000 & $\begin{array}{c}0.487 \\
(0.247)\end{array}$ & & & & & & & & & $\begin{array}{c}0.493 \\
(0.229)\end{array}$ \\
\hline $2 \& 0.7$ & 1.690 & $\begin{array}{c}0.416 \\
(0.196)\end{array}$ & $\begin{array}{c}0.405 \\
(0.208)\end{array}$ & $\begin{array}{c}0.371 \\
(0.177)\end{array}$ & $\begin{array}{c}0.372 \\
(0.176)\end{array}$ & $\begin{array}{c}0.368 \\
(0.168)\end{array}$ & $\begin{array}{c}0.352 \\
(0.170)\end{array}$ & $\begin{array}{c}0.348 \\
(0.194)\end{array}$ & $\begin{array}{c}0.345 \\
(0.163)\end{array}$ & $\begin{array}{c}0.386 \\
(0.172)\end{array}$ & $\begin{array}{c}0.432 \\
(0.207)\end{array}$ \\
\hline $2 \& 0.8$ & 1.581 & & & & & & & & & & $\begin{array}{c}0.378 \\
(0.186)\end{array}$ \\
\hline $3 \& 0.2$ & 3.873 & $\begin{array}{l}1.065 \\
(0.424)\end{array}$ & $\begin{array}{c}0.939 \\
(0.351)\end{array}$ & $\begin{array}{c}0.827 \\
(0.382)\end{array}$ & $\begin{array}{c}0.805 \\
(0.322)\end{array}$ & $\begin{array}{c}0.907 \\
(0.416)\end{array}$ & $\begin{array}{c}0.896 \\
(0.373)\end{array}$ & $\begin{array}{c}0.769 \\
(0.298)\end{array}$ & $\begin{array}{c}0.788 \\
(0.331)\end{array}$ & $\begin{array}{c}0.924 \\
(0.351)\end{array}$ & $\begin{array}{c}0.949 \\
(0.406)\end{array}$ \\
\hline $3 \& 0.3$ & 3.162 & $\begin{array}{c}0.781 \\
(0.365)\end{array}$ & $\begin{array}{c}0.717 \\
(0.281)\end{array}$ & $\begin{array}{c}0.641 \\
(0.263)\end{array}$ & $\begin{array}{c}0.661 \\
(0.264)\end{array}$ & $\begin{array}{c}0.710 \\
(0.300)\end{array}$ & $\begin{array}{c}0.679 \\
(0.311)\end{array}$ & $\begin{array}{c}0.631 \\
(0.278)\end{array}$ & $\begin{array}{c}0.627 \\
(0.263)\end{array}$ & $\begin{array}{c}0.705 \\
(0.282)\end{array}$ & $\begin{array}{c}0.812 \\
(0.415)\end{array}$ \\
\hline $3 \& 0.5$ & 2.450 & $\begin{array}{c}0.619 \\
(0.257)\end{array}$ & $\begin{array}{c}0.613 \\
(0.283)\end{array}$ & $\begin{array}{c}0.527 \\
(0.206)\end{array}$ & $\begin{array}{c}0.506 \\
(0.212)\end{array}$ & $\begin{array}{c}0.519 \\
(0.218)\end{array}$ & $\begin{array}{c}0.565 \\
(0.274)\end{array}$ & $\begin{array}{c}0.509 \\
(0.221)\end{array}$ & $\begin{array}{c}0.512 \\
(0.227)\end{array}$ & $\begin{array}{c}0.566 \\
(0.263)\end{array}$ & $\begin{array}{c}0.626 \\
(0.261)\end{array}$ \\
\hline $3 \& 0.7$ & 2.070 & $\begin{array}{c}0.537 \\
(0.211)\end{array}$ & $\begin{array}{c}0.500 \\
(0.205)\end{array}$ & $\begin{array}{c}0.450 \\
(0.197)\end{array}$ & $\begin{array}{c}0.464 \\
(0.212)\end{array}$ & $\begin{array}{c}0.447 \\
(0.200)\end{array}$ & $\begin{array}{c}0.443 \\
(0.189)\end{array}$ & $\begin{array}{c}0.430 \\
(0.194)\end{array}$ & $\begin{array}{c}0.457 \\
(0.205)\end{array}$ & $\begin{array}{c}0.516 \\
(0.215)\end{array}$ & $\begin{array}{c}0.524 \\
(0.229)\end{array}$ \\
\hline $3 \& 0.8$ & 1.937 & $\begin{array}{c}0.534 \\
(0.212)\end{array}$ & $\begin{array}{c}0.476 \\
(0.199)\end{array}$ & $\begin{array}{c}0.450 \\
(0.188)\end{array}$ & $\begin{array}{c}0.453 \\
(0.199)\end{array}$ & $\begin{array}{c}0.441 \\
(0.191)\end{array}$ & $\begin{array}{c}0.423 \\
(0.179)\end{array}$ & $\begin{array}{c}0.439 \\
(0.212)\end{array}$ & $\begin{array}{c}0.423 \\
(0.186)\end{array}$ & $\begin{array}{c}0.466 \\
(0.195)\end{array}$ & $\begin{array}{c}0.538 \\
(0.284)\end{array}$ \\
\hline
\end{tabular}

\section{Results}

\subsection{Quantifying the Differences of All Ten Fingers with Characteristics of the Original Meyer Model}

\subsubsection{Differences in Reaction Time}

In order to facilitate comparative analysis with previous similar literature [5,27], we first analyzed the difference in average reaction times between symmetrical fingers across all IDs. For symmetrical fingers, the mean values of $T_{R}$ for "long" fingers (LI, LM, RI, and RM) were about 40-50 ms faster than that for "middle" fingers (LT, LR, RT, and RR). It was also found that the mean values of $T_{\mathrm{R}}$ for the "middle" fingers were 50-60 ms quicker than that for "short" fingers (LL and RL). But the mean values of $T_{\mathrm{R}}$ for fingers on the right hand were about $11 \mathrm{~ms}$ quicker than that for fingers on the left hand.

A two-way ANOVA was conducted to analyze the effects of subjects and their fingers on the mean values of $T_{R}$ across all the combinations. The casewise diagnostics test showed that seven samples had the largest errors and might be abnormal. As a behavioral measurement, we tended to be less conservative so that the samples with large residuals remained. Tests of between-subjects showed that there were interactions between the effect of subjects and fingers $(F(99,1800)=1.913, p<0.05)$. Likewise, a two-way ANOVA with the factors of hands and A and W combinations was conducted to analyze their effects on the mean values of $T_{R}$ across all subjects. The casewise diagnostics test showed that two samples had the largest errors and might be abnormal. Tests of between-subjects showed that there were no interactions between the effect of subjects and fingers $(F(13,112)=0.229$, $p>0.05)$. Subsequently, tests of simple main effects showed that both the subjects $(F(11,1800)=97.452$, $p<0.05)$ and the fingers $(F(9,1800)=20.014, p<0.05)$ had a significant effect on the reaction time. 
Bonferroni pair-wise comparisons were used to follow up significant main effects and simple main effects. Tests of main effects showed that no significant effect of hands on the reaction time was found $(F(1,112)=1.843, p>0.05)$, while there was a significant effect of $A$ and $W$ combinations on the reaction time $(\mathrm{F}(13,112)=1.843, p<0.05)$. There was no difference between symmetrical fingers on two hands. The differences in $T_{\mathrm{R}}$ among the "long" fingers were not significant $(p>0.05)$. A similar conclusion was found among the "middle" fingers and the "short" fingers.

\subsubsection{Differences in Index of Performance}

According to Fitts' law, the reaction time $T_{\mathrm{R}}$ is a linear function of the IDs in reciprocal tapping tasks. Compared to the $T_{R}$, the value of $I P$ would be approximately constant regardless of how much the values of $I D$ were. In accordance with previous work [36], the IP was still employed as the behavioral metric for the quantifiable and comparable difference of capability among individual fingers in accurate discrete force control. Several important characteristics in the original Meyer model are shown in Table 2 for each individual finger. Moreover, the order of the fingers was arranged symmetrically to facilitate comparison between two hands. The values of $I P$ for the "long" fingers were greater than that for the "middle" fingers, and the values of $I P$ for the "middle" fingers were greater than that for the "short" fingers. Generally, IP values of fingers on the right hand were greater than that of fingers on the left hand except for the left index finger. The quantitative differences of IPs among ten fingers were generally consistent with the hierarchy in $T_{R}$.

Table 2. Characteristics of the original Meyer model for individual fingers.

\begin{tabular}{cccccc}
\hline Hand & Fingers & $\boldsymbol{a}$ & $\boldsymbol{b}$ & $\boldsymbol{R}^{\mathbf{2}}$ & $\boldsymbol{I P}$ (Bits/s) \\
\hline \multirow{6}{*}{ Left } & Little & 0.0044 & 0.2542 & 0.9632 & 3.9343 \\
& Ring & 0.0495 & 0.2165 & 0.9602 & 4.6193 \\
& Middle & 0.0659 & 0.1886 & 0.9624 & 5.3022 \\
& Index & 0.1078 & 0.1755 & 0.9243 & 5.6984 \\
& Thumb & 0.0480 & 0.2145 & 0.9171 & 4.6625 \\
\hline \multirow{6}{*}{ Right } & Thumb & 0.0466 & 0.2112 & 0.9168 & 4.7344 \\
& Index & 0.0704 & 0.1829 & 0.9471 & 5.4685 \\
& Middle & 0.0726 & 0.1834 & 0.9412 & 5.4530 \\
& Ring & 0.0347 & 0.2225 & 0.9751 & 4.4943 \\
& Little & 0.0444 & 0.2400 & 0.9639 & 4.1672 \\
\hline
\end{tabular}

\subsubsection{Differences in Goodness of Fit}

All values of the goodness of fit $R^{2}$ were more than 0.9 . The ranking order of the goodness of fit $R^{2}$ was almost the opposite of that of $I P$. The ranking order of the goodness of fit among five fingers of one hand was as follows: $R^{2}$ was the greatest for the "short" fingers, followed by the "middle" fingers except for the right ring finger, and then the "long" fingers respectively. In contrast, the right index finger indicated the greatest value of $I P$, while it was not as optimal as the right little finger with respect to the goodness of fit. In comparison, the little fingers on both hands were quite consistent with linear function in Fitts' law in terms of the goodness of fit, even though it showed the lowest value of IP.

\subsection{Quantifying Differences of All Ten Fingers with a Modified Meyer Model and Its' Characteristics}

\subsubsection{Modified Meyer Model}

It is indeed true that quantifying input systems can be made by measuring $\boldsymbol{a}$ and $\boldsymbol{b}$ parameters in the complete Fitts' law. Intercept $\boldsymbol{a}$ reflects the noninformational aspect and slope $\boldsymbol{b}$ reflects the informational aspect of input performance. Zhai et al. [35] systematically demonstrated the shortcomings of the traditional definitions of throughput in Fitts' law, and thus recommending its two coefficients to characterize an input performance. Therefore, it was very important to report values 
of both $\boldsymbol{a}$ and $\boldsymbol{b}$, otherwise, the results from Zhai and co-workers cannot be generalized beyond the specific experimental target distances and sizes. However, usage of the two characteristics $\boldsymbol{a}$ and $\boldsymbol{b}$ was not convenient for directly comparing the differences of each finger. For the sake of convenience, a composite variable integrating the two coefficients would be quite useful to simplify the quantification and comparison between different fingers. According to values in Table 2, Figure 3 showed the scatter plot of coefficients $\boldsymbol{a}$ and $\boldsymbol{b}$ for each of the ten fingers. For all fingers, $\boldsymbol{a}$ and $\boldsymbol{b}$ showed an almost symmetrical distribution in both vertical and horizontal directions, except that little fingers were slightly different. Accordingly, a conclusion that could be drawn from the distributions of $\boldsymbol{a}$ and $\boldsymbol{b}$ was that there might be an implied symmetrical axis along the horizontal axis, regardless of their physical units given from the information-theoretic perspective. In a fitting linear regression to examine this potential conclusion, a novel finding was that the sum of two coefficients in the original Meyer model was approximately constant across ten fingers. As a result, an alternative linear function $(\boldsymbol{a}+\boldsymbol{b}=0.2633$, $R^{2}=0.81$ ) was discovered, supporting the conclusion at least moderately well. Based on these results above, we simplified the original Meyer model of SAT into a novel model with only one integrated coefficient, facilitating the intuitive comparison among all ten fingers. The modified regression function was formulated as:

$$
T_{\mathrm{R}}=0.2633+b \cdot(I D-1)
$$

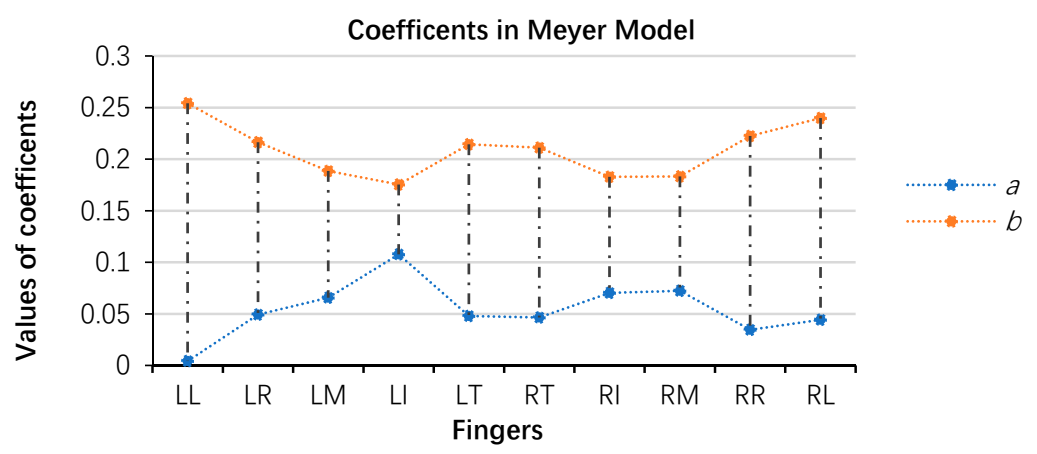

Figure 3. Values of $\boldsymbol{a}$ and $\boldsymbol{b}$ for all ten fingers.

\subsubsection{Index of Performance and Goodness of Fit in the Modified Meyer Model}

Several important characteristics of the modified Meyer model are also shown in Table 3 for individual fingers. Table 3 summarizes a similar ranking order of the values of $I P$ and $R^{2}$ for individual fingers except for the left index finger. However, the value of $I P$ of the left index finger was greater than that of the left middle finger in the original Meyer model, while the logical relationship between the two fingers was opposite in the modified model. The goodness of fit $\left(R^{2}\right)$ showed that the modified Meyer model was also able to fit values of $T_{R}$ for all fingers perfectly $\left(R^{2}>0.9\right)$. Although the goodness of fit of the modified model for each finger was slightly worse, the change was not statistically significant $(\mathrm{t}(9)=-2.2431, p>0.05)$. Therefore, the values of $R^{2}$ in Tables 2 and 3 showed that the modified Meyer model in Equation (2) was, to some extent, as good as the original Meyer model in Equation (1).

\subsubsection{Regression Analysis in the Modified Meyer Model}

To avoid the poor distinction between graphics caused by the overlap of all ten fingers, Figure 4 illustrates linear regression lines by leveraging the modified Meyer model for each individual finger for both hands. Same colored markers in the scatter plot represented the observed values of $T_{R}$ for one of five fingers on the left or right hand across $\mathrm{A}$ and $\mathrm{W}$ combinations. The solid lines represented the fit of predictions made by the modified Meyer model. The two lines of the index and middle fingers were so close that they almost overlapped. Observing specifically how well the distributions of $T_{R}$ adhered to the linear model across IDs, we found that markers of $T_{\mathrm{R}}$ were all slightly above or below their respective regression lines under several IDs. 
Table 3. Characteristics of the modified Meyer model for individual fingers.

\begin{tabular}{ccccc}
\hline Hand & Fingers & $\boldsymbol{b}$ & $\boldsymbol{R}^{\mathbf{2}}$ & $\boldsymbol{I P}$ (bits/s) \\
\hline \multirow{6}{*}{ Left } & Little & 0.2513 & 0.9630 & 3.9788 \\
& Ring & 0.2181 & 0.9602 & 4.5847 \\
& Middle & 0.1832 & 0.9613 & 5.4576 \\
& Index & 0.1876 & 0.9180 & 5.3296 \\
& Thumb & 0.2140 & 0.9171 & 4.6737 \\
\hline \multirow{4}{*}{ Right } & Thumb & 0.2079 & 0.9165 & 4.8108 \\
& Index & 0.1768 & 0.9456 & 5.6574 \\
& Middle & 0.1789 & 0.9404 & 5.5892 \\
& Ring & 0.2188 & 0.9747 & 4.5709 \\
& Little & 0.2528 & 0.9599 & 3.9561 \\
\hline
\end{tabular}

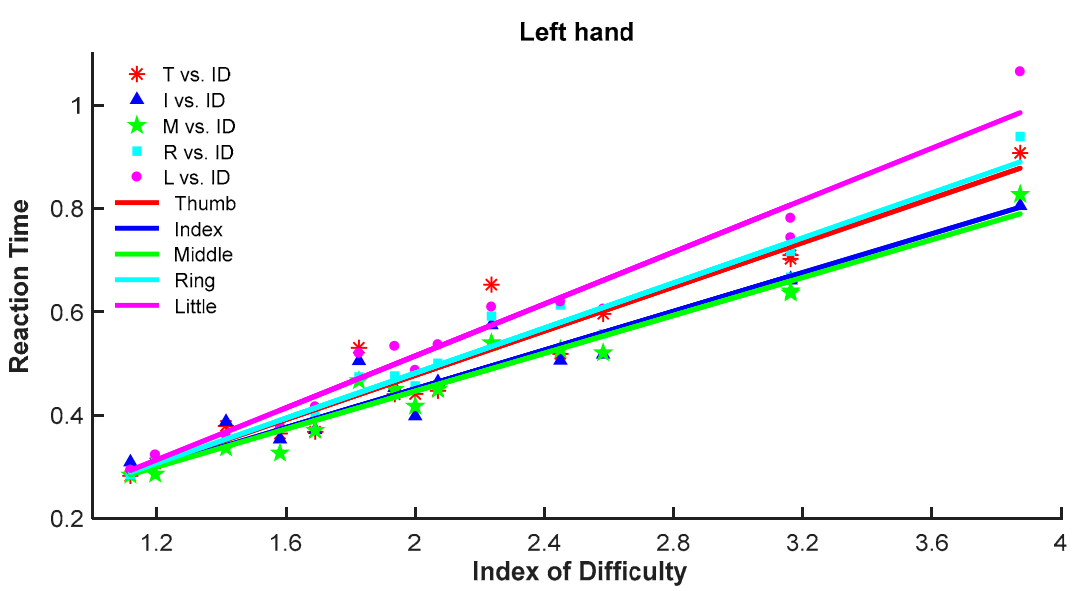

(a)

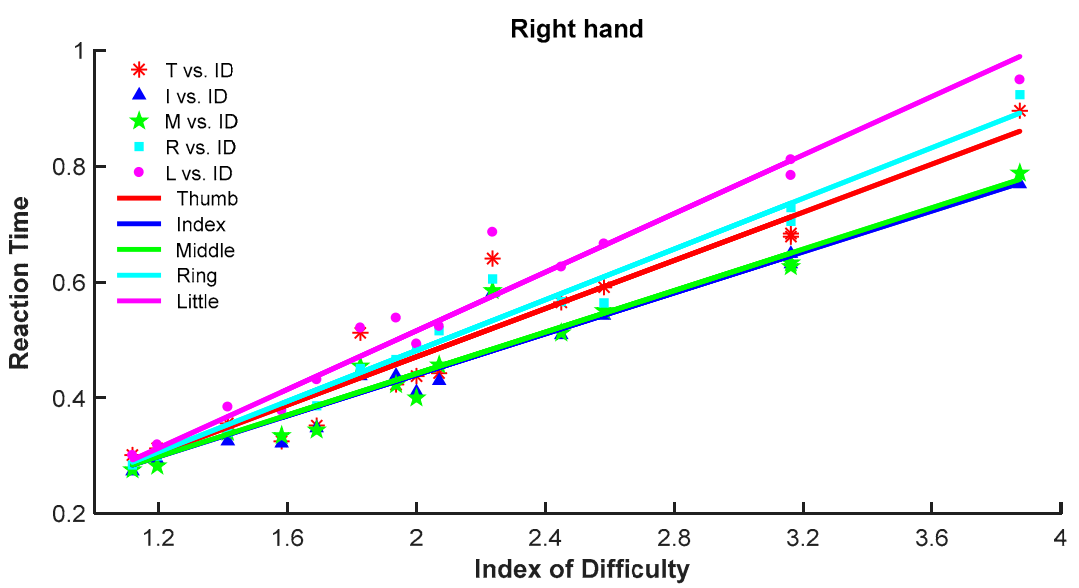

(b)

Figure 4. Linear fitting results for individual fingers leveraging the Meyer model. (a) Linear fitting for fingers on the left hand. (b) Linear fitting for fingers on the right hand.

Accordingly, the observed values of $T_{\mathrm{R}}$ obviously deviated from the predicted values of the Meyer model for several A and W combinations. To highlight these combinations, Figure 5 illustrates a color topography for margins of the $T_{\mathrm{R}}$ by subtracting the predicted values from the observed values. When the magnitude of the target force was $1 \mathrm{~N}$ or $3 \mathrm{~N}$ in given combinations, the reaction time of the subjects in the actual force control task tended to be greater than that predicted by the Meyer model, while the reactions tended to cost less time for combinations when the magnitude of target forces was $2 \mathrm{~N}$. 


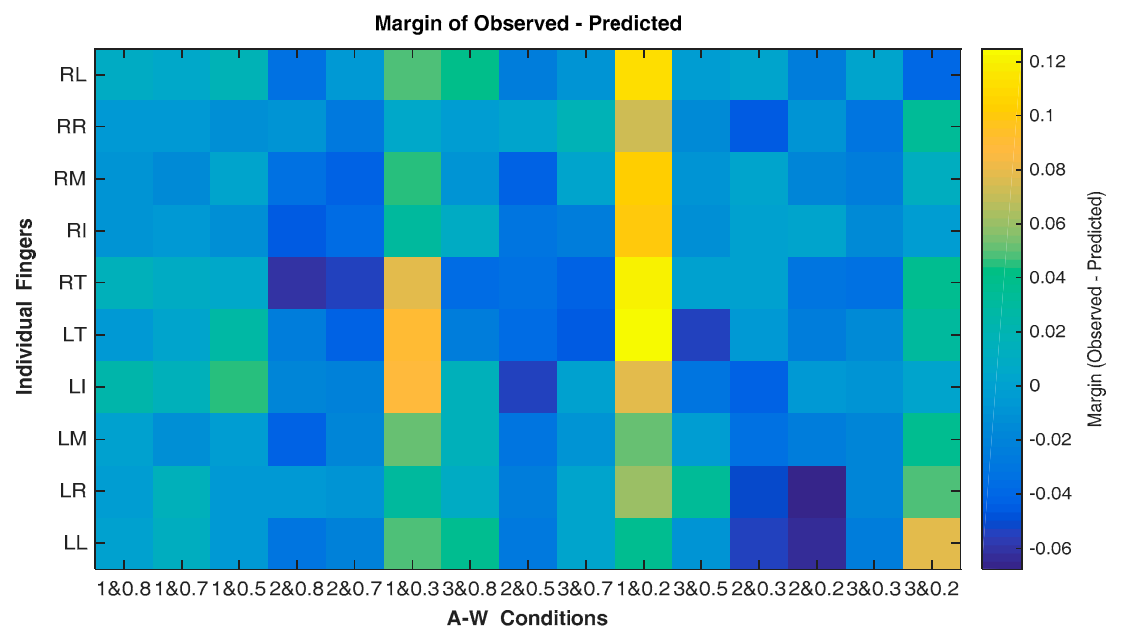

Figure 5. Margins between observed and predicted values.

\subsubsection{Paired Comparison among all Ten Fingers}

Taking the IP in the modified Meyer model as a behavioral biometric, we quantified and scaled the force control capacities over a set of IDs among all ten fingers by the paired comparison of $I P$, as shown in Table 4. Accordingly, the right index finger, right middle finger, and the left index were used as a benchmark, respectively, to compare force control capacities among four symmetrical fingers employed in previous studies $[17,18]$. Therefore, force control capacities of the other fingers were scaled as following: $I P_{\mathrm{LI}}=0.9421 I P_{\mathrm{RI}}, I P_{\mathrm{RM}}=0.988 I P_{\mathrm{RI}}, I P_{\mathrm{LI}}=0.9765 I P_{\mathrm{LM}}$, and $I P_{\mathrm{LM}}=0.9765 I P_{\mathrm{RM}}$. Consequently, the capabilities of the symmetrical index fingers differed greatly, but the differences between the other three fingers were small.

Table 4. Pairwise comparisons of the index of performance $(I P)$ among all ten fingers.

\begin{tabular}{ccccccccccc}
\hline Fingers & LL & LR & LM & LI & LT & RT & RI & RM & RR & RL \\
\hline LL & 1.0000 & 1.1523 & 1.3717 & 1.3395 & 1.1746 & 1.2091 & 1.4219 & 1.4047 & 1.1488 & 0.9943 \\
LR & 0.8678 & 1.0000 & 1.1904 & 1.1625 & 1.0194 & 1.0493 & 1.2340 & 1.2191 & 0.9970 & 0.8629 \\
LM & 0.7290 & 0.8401 & 1.0000 & 0.9765 & 0.8564 & 0.8815 & 1.0366 & 1.0241 & 0.8375 & 0.7249 \\
LI & 0.7466 & 0.8602 & 1.0240 & 1.0000 & 0.8769 & 0.9027 & 1.0615 & 1.0487 & 0.8577 & 0.7423 \\
LT & 0.8513 & 0.9810 & 1.1677 & 1.1403 & 1.0000 & 1.0293 & 1.2105 & 1.1959 & 0.9780 & 0.8465 \\
RT & 0.8271 & 0.9530 & 1.1345 & 1.1078 & 0.9715 & 1.0000 & 1.1760 & 1.1618 & 0.9501 & 0.8223 \\
RI & 0.7033 & 0.8104 & 0.9647 & 0.9421 & 0.8261 & 0.8504 & 1.0000 & 0.9879 & 0.8080 & 0.6993 \\
RM & 0.7119 & 0.8203 & 0.9765 & 0.9535 & 0.8362 & 0.8607 & 1.0122 & 1.0000 & 0.8178 & 0.7078 \\
RR & 0.8705 & 1.0030 & 1.1940 & 1.1660 & 1.0225 & 1.0525 & 1.2377 & 1.2228 & 1.0000 & 0.8655 \\
RL & 1.0057 & 1.1589 & 1.3796 & 1.3472 & 1.1814 & 1.2161 & 1.4300 & 1.4128 & 1.1554 & 1.0000 \\
\hline
\end{tabular}

Furthermore, differences in the SAT between symmetrical fingers on both hands have not been fully revealed during repetitive discrete force control tasks. As a result, it would be also very necessary and valuable for pairings of responding fingers to quantify and compare the force control capacities among ten fingers. Thus, pairwise comparisons showed that the differences between symmetrical two fingers on both hands were small except for the pair of two index fingers according to Table 4.

\section{Discussion}

The results of quantifying the differences among the fingers of the right hand with $T_{R}$ are generally consistent with those of previous studies, although the experimental settings were slightly different. That is, there were measurable differences in the reaction time among individual fingers of the right hand. Aoki et al. [2] observed differences in the dynamic motor function among the four fingers except for the thumb of the dominant hand under a rapid repetitive tapping condition. The thumb was also 
checked in this study since its' widely used to operate remote controllers, gamepads, and smartphones, especially when gripping these devices with one hand. In the experimental tasks proposed by Aoki et al. [2], key-contact forces for all fingers and the movement velocity of the tapping finger were monitored. In terms of the movement velocity, Aoki et al. [2] found that the index finger attained the fastest cadence and greatest movement velocity, followed by the middle, little, and ring fingers, respectively. This finding was generally consistent with our results obtained in ranking the orders of $T_{R}$. Analyses of Aoki et al. [2] mainly focused on the speed and enslaving effects in multi-finger force production [20], rather than the force control capability in the speed-accuracy trade-off. In a study of speed-accuracy trade-offs during finger force production, Gorniak et al. [27] argued that the intervals between total force maxima and minima would not accurately reflect actual movement time. Because they found that some subjects exhibited a dwell time at the targets during a cyclic force-production task. Thus, for the finger force data, the oscillations between targets were considered to consist of two components: an increase of, and decrease of the total force [27]. Movement times for the two components were averaged separately across each trial. Gorniak et al. [27] found there was no difference in movement time for the force-increase component among the single-finger tasks across ID levels. However, there was a difference in movement time the for force-decrease component among the single-finger tasks. For the right hand, the movement time for force-decrease component of the index and middle fingers was about $50 \mathrm{~ms}$ quicker than both the ring and little fingers. Furthermore, the movement time for the force-decrease component of the middle finger of the right hand was $33 \mathrm{~ms}$ less than the middle finger of the left hand. The results from Gorniak et al. were also in line with our findings when indexed by the $T_{R}$. Therefore, the experimental tasks in these two previous studies could be considered as two sub-movement segments of our discrete finger force control process in each trial.

In addition to the reaction time $T_{\mathrm{R}}$, we also employed $I P$ to quantify and rank the force control capabilities among individual fingers on both hands. As an indicator of reaction speed, reaction time or movement time is not an ideal index to measure the capability of the input system itself, because it is a function that involves both task-related parameters and input system-related parameters according to Fitts' law. Due to signal-dependent noise in the neural control signal, the reduction of movement time in a given motor task increases spatial variability [26]. Conversely, the reduction of spatial variability increases movement time. Therefore, the IP has been widely used as one of behavioral metrics from Fitts' law to quantify the information processing capacity of the subjects [26]. ISO 9241-9 Annex B recommended using the index of performance (IP) as the sole metric of input device performance [39]. Consequently, IP had also proved to be an effective indicator for quantifying finger differences in an earlier pilot study [36]. In the current experiments of repetitive discrete force control tasks, the ranking order of $I P \mathrm{~s}$ for fingers appeared to be consistent with that revealed by the reaction time. According to $I P$, the throughput [35] was greatest for the index finger, followed by the middle, thumb, ring, and little fingers respectively. It should be noted that the effect of the individual differences of subjects on $I P$ measurements could no longer be ignored. On the one hand, it would be better if more users were recruited in future related assessments and applications. On the other hand, even if some fingers were found to be different according to the individual, the method in this paper might still be instructive, providing the individual differences that could be determined before the users begin his work.

Moreover, the pending issue mentioned in Section 1 could be solved by the pairwise comparisons of $I P$ among all ten fingers, as shown in Table 4 . These results would provide intuitive guidance for application design involving the pressing operations of two fingers. The answer to the selection of two fingers pairs from all ten fingers could be concluded as follows: the RI and RM would be the best-paired fingers if two responding fingers were required to be on one hand; two symmetrical fingers except for symmetrical index fingers would be the best-paired fingers if two responding fingers were required to be on different hands. The complex finger pairing between hands and fingers interaction indicated that it was difficult to generalize the reaction-time pattern found for a given pair of fingers in a two-choice reaction task to any two-finger choice situation [40]. The pattern of reaction times 
in two-finger choice reaction tasks appeared to be very sensitive to experimental factors such as the response-pair uncertainty, foreperiod duration, hand placement, or finger placement [40]. It should be noted that discrepant results between several previous studies could come from different preparation strategies used by the subjects, and the experimental designs $[4,41]$.

Because of the different measurement tasks and metrics used in our experiments, the order between the ring and little fingers in this rank was indeed slightly different from that revealed by previous observations $[2,5]$. There was no significant difference between symmetrical fingers on two hands from the respective of $T_{R}$, even though the right thumb, index, and middle fingers were slightly superior to its symmetrical counterparts of the left hand in terms of $I P$, as shown in Table 3, while this phenomenon did not appear on the other fingers. However, Gatewood justified that the fingers of the right hand were, on average, faster than the fingers of the left hand [5]. The fingers of the right hand also surpassed that of the left hand in accuracy, but such differences were slight. This finding could be explained by the fact that all subjects were right-handed in his experiments. Aoki and co-workers speculated that the lower cadence achieved by the ring finger might be attributed more to a lack of independence at the level of voluntary neuromuscular control, than to innate mechanical interaction with the other fingers [2]. Aoki et al. [2] presumed that numerous factors collectively contributed to the kinematic and kinetic differences observed. Therefore, our observation that the ring fingers are inherently superior to the little fingers may be attributed to two main aspects: tasks settings and behavioral metrics. The repetitive discrete force control task in this paper was the classic Fitts' reciprocal tapping task that required a tapping reaction with specific accuracy or speed constraints, and wherein the performance was quantified by the IP. Referred to similar research context to previous investigators [27,29], we also primarily consider behavioral data, and to elucidate them here even though we also recognize the importance of biomechanical and neurophysiological evidence when regarding different movement performance. The evidence already suggested that the individual finger movement control was distributed widely in the human primary motor cortex hand area [42] and produced by the activity of several muscles [43]. Herein, a more insightful study from Szychlinska et al. [44] is recommendable owing to the perspective of hand anatomy and biomechanics, helping to understand the neurobiological mechanisms underlying the differentiation among fingers.

All characteristics of Fitts' law formula have their own advantages, which should be selected according to the specific application requirements. The difference among fingers was not only reflected in $T_{\mathrm{R}}$ and $I P$, but also in the goodness of fit $R^{2}$. Table 3 showed that the ranking order of $T_{\mathrm{R}}$ was consistent with $I P$ among fingers, while the ranking order of $I P$ was inconsistent with the goodness of fit $R^{2}$ among fingers. Specifically, the ranking orders of $T_{\mathrm{R}}$ and $I P$ indicated that the index finger possessed the fastest reaction speed and the largest force control capacity. Whereas, the ranking order of $R^{2}$ showed that the little fingers had better conformities with Fitts' law, which suggested that reaction times of the little fingers possessed the optimal predictable accuracy in the repetitive discrete force control. Meyer et al. [29] compared the observed ratios of the mean primary-sub-movement durations with the predicted ones and found the fit was good only for the relatively difficult target combinations. Accordingly, the findings from Meyer et al. [29] to some extent provided a good account for our observation that the goodness of fit for the little finger was quite excellent among fingers on one hand. Compared with the index fingers, it was relatively difficult for the little finger to react quickly in all $\mathrm{A}$ and $\mathrm{W}$ combinations. This was in accord with Aoki's explanation of the relationship between force production capacity and involved muscle [2]. Admittedly, IP was still a more important metric if more emphasis was placed on the SAT in multi-finger inputs, but goodness of fit would be more suitable for a finger force control task requiring accurate repeatable reaction over various difficulties. Therefore, leveraging the differences of $I P$ among all ten fingers, the false recognition rate of the little finger may be lower because of its higher goodness of fit relative to the index finger. This hypothesis needs to be further confirmed after sufficient samples and suitable pattern recognition methods have been completed in the future work. 
With the modified Meyer model described in Equation (2), just one trial was enough to work out the IP of each finger. Admittedly, this innovative finding facilitated the ability to quantify and compare the motor capabilities among all ten fingers in the SAT tasks, but it also led to a newly emerging issue. For one thing, the sum of $\boldsymbol{a}$ and $\boldsymbol{b}$ was a constant 0.2633 in the modified Meyer model. This constant could not be interpreted from the classic information-theoretic perspective due to the different units of coefficients $\boldsymbol{a}(\mathrm{s})$ and $\boldsymbol{b}$ (s/bits). Also, the constant component of reaction time suggested that there was a sub-movement or cognitive sub-process whose duration was not significantly different among all ten fingers from the perspective of sub-movements [29,45]. Even though Gorniak et al. [27] found there was no difference among single-finger tasks in the movement time for the force increase component, there was still insufficient evidence to extend their conclusions from cyclic force control to current discrete force control tasks. Christe et al. [26] stated that speed constraints generated gains in the temporal domain and costs in the spatial one, while spatial constraints generated gains in the spatial domain and costs in the temporal one; finally, sequential constraints revealed the integrative nature of the cognitive operations involved in motor production. Goodgold-Edwards et al. [32] advocated future studies would be required to describe cognitive strategies of children with musculoskeletal and neuromuscular dysfunction and to examine the effects of teaching superior cognitive strategies on performance during meaningful tasks. Therefore, the interpretation for the constant 0.2633 would be a pending issue and required to be widely explored in the future works.

The modified Meyer model was not yet able to accurately predict reactions for a few initial-boundary difficulty levels in discrete finger force control. A similar fitting error was observed in a previous investigation in which the Meyer model overestimated the observed values for easy targets and underestimated them for difficult targets [45]. Li et al. [28] confirmed that the fingertip force control process obeyed Fitts' law only if the index of difficulty (ID) was within a specific range. Three common formulas of the speed-accuracy trade-off model were considered not to be suitable anymore for a larger $I D$, and it is necessary to explore other new formulas [45]. It was supposed that the difficulty of this level had already approached the human limit for accuracy of controlling muscle. Meyer et al. [45] argued that human performance might fluctuate randomly due to the existence of noise in the neuromotor system. The linear trade-off and the stochastic mechanisms underlying it constituted basic psychophysical characteristics of biological motor systems. These characteristics placed fundamental limitations on how people might perform other types of movement tasks. Accordingly, our hypothesis is that the differences in force control among fingers are the reflection of its intrinsic capabilities, which are determined by the biomechanics and anatomical structure of individual fingers. Some evidence of the noise underlying such variability could be seen, for example, in records of electromyographic (EMG) activity during rapid movements, which typically exhibited agonist and antagonist bursts that fluctuated substantially from trial to trial [29]. As a result, all combinations with target force magnitude of $4 \mathrm{~N}$ were no longer employed, and additional target tolerances of 0.2 and $0.3 \mathrm{~N}$ were added in current experiment. Moreover, the observed values for relatively small or large magnitude of force tended to be underestimated. It was possible that when the target force magnitude was small (i.e., $1 \mathrm{~N}$ ), the starting point and the end point were close, which made visual-induced target perception difficult. When the target force magnitude was large, muscle spasm might cause greater neuromotor noise. Hence, the force magnitude $2 \mathrm{~N}$ with tolerance $0.2 \mathrm{~N}$ was a recommendable $\mathrm{A}$ and $\mathrm{W}$ combination for difficulty of accurate force control task when selecting task difficulties for prediction or finger identification in the future.

Quantified differences among all ten fingers in the SAT for force control could also be a keystroke dynamics identity, providing alternative guidance for interaction design. For example, Findlater et al. [8] used simulations of touchscreen typing data to show that a personalized input model for ten-finger typing greatly improved key-press classification accuracy. However, Findlater et al. primarily focused on ten-finger typing features consisting of touch locations, touch areas, and movement times, while the force control differences in the process of touching keys were not fully exploited. Pressure-based interaction allowed adding features ranging from the pressure of the screen or finger area to the 
classical time-based features used for keystroke dynamics. Results from Antal et al. [46] showed that these additional features enhance the accuracy of both processes. Hereby, these studies suggested a heuristic supporting the pattern analysis and recognition of tapping fingers by checking responding finger of each typing, similar to fingerprint identification [47,48]. Keystroke dynamics of finger force control for user authentication has become an active research area due to its low cost and ease of integration with existing security systems. Orozco et al. [49] designed a haptic system in which position, velocity, force, and torque data from the tool was continuously measured and stored as users were performing a specific task. Subsequently, several algorithms and methods were developed to extract biometric features from the measured data. Overall, the results suggested reasonable practicality of implementing haptic-based biometric systems, which was an avenue worth pursuing. As one of behavioral biometrics, comparative differences in the performance of SAT may be instructive in finger identification, diversifying keyboard-interactive styles in identity authentication [50].

\section{Conclusions}

In this paper, we studied the force control capabilities of all ten fingers in SAT tasks involving discrete force control. The SAT performance of five fingers of the right hand was confirmed to follow the Meyer model. We found that the five fingers of the left hand also obeyed the Meyer model in the SAT performance. Moreover, all ten fingers differed in reaction time, the index of performance (IP), and the goodness of fit $R^{2}$. The index or middle fingers have a shorter reaction time, suggesting that they are the better choice for applications requiring faster response. The little fingers are better in the goodness of fit $R^{2}$ than other fingers. This distinction implies that for applications requiring more accurate response time they are the better choice.

A modified Meyer model was proposed to characterize individual fingers, which reduces the number of parameters in the original Meyer model from two to one. This dimensional reduction makes the parameter estimation of the model much easier and simplifies the prediction of reaction time using the model. Pairwise comparisons using the modified Meyer model revealed that the differences between symmetrical two fingers on both hands were insignificant, except for the pair of two index fingers.

As one of potential behavioral biometrics, the differences among all ten fingers in the force control capability are instructive in engineering design for tasks requiring quick and repetitive force control using multiple fingers. One of our future works is to develop highly efficient algorithms for finger pattern recognition using the above quantitative measure of the difference. Additionally, the quantitative differences of individual fingers could also support clinical assessments of finger motor dysfunction for patients with upper motoneuron lesions [22,51,52] or Parkinson's disease [53,54].

Author Contributions: C.P. and D.W. conceived the idea, C.P. conducted the experiments and wrote the initial draft of the paper, and D.W. and Y.Z. revised the manuscript. All authors read and approved the manuscript.

Funding: This work was supported by the National Key Research and Development Program under Grant 2017YFB1002803, and in part by the National Natural Science Foundation of China under Grant 61572055.

Conflicts of Interest: The authors declare no conflict of interest.

\section{References}

1. Jones, L.A.; Lederman, S.J. Human Hand Function; Oxford University Press: New York, NY, USA, 2006; pp. 131-149.

2. Aoki, T.; Francis, P.R.; Kinoshita, H. Differences in the abilities of individual fingers during the performance of fast, repetitive tapping movements. Exp. Brain Res. 2003, 152, 270-280. [CrossRef]

3. Billon, M.; Bootsma, R.J.; Mottet, D. The dynamics of human isometric pointing movements under varying accuracy requirements. Neurosci. Lett. 2000, 286, 49-52. [CrossRef]

4. Kornblum, S. Response competition and/or inhibition in two-choice reaction time. Psychon. Sci. 1965, 2, 55-56. [CrossRef] 
5. Gatewood, E.L. Individual differences in finger reactions. Psychol. Monogr. Gen. Appl. 1920, $28,1-43$. [CrossRef]

6. Bhatnagar, M.; Jain, R.K.; Khairnar, N.S. A Survey on Behavioral Biometric Techniques: Mouse vs. Keyboard Dynamics. Int. J. Comput. Appl. 2013, 975, 27-30.

7. Martin, B.J.; Natarajan, S.; Klinenberg, E.; Serina, E.; Armstrong, T.J.; Foulke, J.A.; Rempel, D. Keyboard Reaction Force and Finger Flexor Electromyograms during Computer Keyboard Work. Hum. Factors J. Hum. Factors Ergon. Soc. 1996, 38, 654-664. [CrossRef] [PubMed]

8. Findlater, L.; Wobbrock, J. Personalized input: Improving ten-finger touchscreen typing through automatic adaptation. In Proceedings of the SIGCHI Conference on Human Factors in Computing Systems, Seattle, WA, USA, 5-10 May 2012; ACM: New York, NY, USA, 2012; pp. 815-824.

9. Zhai, S.; Hunter, M.; Smith, B. Performance Optimization of Virtual Keyboards. Hum.-Comput. Interact. 2002, 17, 229-269.

10. Sears, A. Improving touchscreen keyboards: Design issues and a comparison with other devices. Interact. Comput. 1991, 3, 253-269. [CrossRef]

11. Corsten, C.; Lahaye, M.; Borchers, J.; Voelker, S. ForceRay: Extending Thumb Reach via Force Input Stabilizes Device Grip for Mobile Touch Input. In Proceedings of the 2019 CHI Conference on Human Factors in Computing Systems, Glasgow, Scotland, UK, 4-9 May 2019; ACM: New York, NY, USA, 2019; pp. 1-12.

12. Zhong, M.; Yu, C.; Wang, Q.; Xu, X.; Shi, Y. ForceBoard: Subtle Text Entry Leveraging Pressure. In Proceedings of the 2018 CHI Conference on Human Factors in Computing Systems, Montreal, QC, Canada, 21-26 April 2018; pp. 1-10.

13. Karrenbauer, A.; Oulasvirta, A. Improvements to keyboard optimization with integer programming. In Proceedings of the ACM Symposium on User Interface Software and Technology, Honolulu, HI, USA, 5-8 October 2014; pp. 621-626.

14. Wu, C.-M.; Smith, S. A haptic keypad design with a novel interactive haptic feedback method. J. Eng. Des. 2015, 26, 169-186. [CrossRef]

15. Bufton, M.J.; Marklin, R.W.; Nagurka, M.L.; Simoneau, G.G. Effect of keyswitch design of desktop and notebook keyboards related to key stiffness and typing force. Ergonomics 2006, 49, 996-1012. [CrossRef]

16. Gerard, M.J.; Armstrong, T.J.; Foulke, J.A.; Martin, B.J. Effects of Key Stiffness on Force and the Development of Fatigue While Typing. Am. Ind. Hyg. Assoc. J. 1996, 57, 849-854. [CrossRef] [PubMed]

17. Moore, A.W.; Gruber, T.; Derose, J.; Malinowski, P. Regular, brief mindfulness meditation practice improves electrophysiological markers of attentional control. Front. Hum. Neurosci. 2012, 6, 1-15. [CrossRef] [PubMed]

18. Peng, C.; Wang, D.; Zhang, Y.; Xiao, J. A Visuo-Haptic Attention Training Game with Dynamic Adjustment of Difficulty. IEEE Access. 2019, 7, 68878-68891. [CrossRef]

19. Latash, M.; Scholz, J.; Danion, F.; Schöner, G. Structure of motor variability in marginally redundant multifinger force production tasks. Exp. Brain Res. 2001, 141, 153-165. [CrossRef] [PubMed]

20. Zatsiorsky, V.M.; Li, Z.-M.; Latash, M.L. Enslaving effects in multi-finger force production. Exp. Brain Res. 2000, 131, 187-195. [CrossRef] [PubMed]

21. Cuadra, C.; Bartsch, A.; Tiemann, P.; Reschechtko, S.; Latash, M.L. Multi-finger synergies and the muscular apparatus of the hand. Exp. Brain Res. 2018, 236, 1383-1393. [CrossRef]

22. Lee, M.; Lee, J.; Shin, J.; Bae, J. Evaluation of Finger Force Control Ability in Terms of Multi-Finger Synergy. IEEE Trans. Neural Syst. Rehabil. Eng. 2019, 27, 1253-1262. [CrossRef]

23. Kjnoshita, H.; Kawai, S.; Ikuta, K. Contributions and co-ordination of individual fingers in multiple finger prehension. Ergonomics. 1995, 38, 1212-1230. [CrossRef]

24. Kong, Y.-K.; Park, H.; Kim, D.; Lee, T.; Roh, E.; Lee, S.; Zhao, W.; Kim, D.-M.; Kang, H.-S. A Study on the Difference of Total Grip Strength and Individual Finger Force between Dominant and Non-dominant Hands in Various Grip Spans of Pliers. J. Ergon. Soc. Korea. 2013, 32, 503-509. [CrossRef]

25. Salmoni, A.W.; Mcllwain, J.S. Fitts' Reciprocal Tapping Task, a Measure of Motor Capacity? Percept. Mot. Sk. 1979, 49, 403-413. [CrossRef]

26. Christe, B.; Burkhard, P.R.; Pegna, A.J.; Mayer, E.; Hauert, C.-A. Clinical Assessment of Motor Function: A Processes Oriented Instrument Based on a Speed-Accuracy Trade-Off Paradigm. Behav. Neurol. 2007, 18, 19-29. [CrossRef] [PubMed]

27. Gorniak, S.L.; Duarte, M.; Latash, M.L. Do synergies improve accuracy? A study of speed-accuracy trade-offs during finger force production. Mot. Control. 2008, 12, 151-172. [CrossRef] 
28. Li, T.; Wang, D.; Peng, C.; Yu, C.; Zhang, Y. Speed-accuracy tradeoff of fingertip force control with visual/audio/haptic feedback. Int. J. Hum.-Comput. Stud. 2018, 110, 33-44. [CrossRef]

29. Meyer, D.E.; Smith, J.E.K.; Kornblum, S.; Abrams, R.A.; Wright, C.E. Speed Accuracy Tradeoffs in Aimed Movements-Toward a Theory of Rapid Voluntary Action. Atten. Perform. 1990, 4, 173-226.

30. Soukoreff, R.W.; MacKenzie, I.S. Towards a standard for pointing device evaluation, perspectives on 27 years of Fitts' law research in HCI. Int. J. Hum.-Comput. Stud. 2004, 61, 751-789. [CrossRef]

31. Irwin, C.B.; Duff, S.N.; Skye, J.L.; Wiegmann, D.A.; Sesto, M.E. Disability and Orientation-Specific Performance during a Reciprocal Tapping Task. In Proceedings of the Human Factors and Ergonomics Society Annual Meeting, San Francisco, CA, USA, 27 September-1 October 2010; pp. 581-585.

32. Goodgold-Edwards, S.A.; Beshere, N.; Murphy, K.; MacNeil, D.; Daoust, B. Cognitive Strategies During a Reciprocal Tapping Task. Phys. Occup. Ther. Pediatr. 1997, 17, 1-19. [CrossRef]

33. Fitts, P.M. The information capacity of the human motor system in controlling the amplitude of movement. J. Exp. Psychol. 1954, 47, 381-391. [CrossRef] [PubMed]

34. Wright, C.E.; Lee, F. Issues Related to HCI Application of Fitts's Law. Hum.-Comput. Interact. 2013, 28, 548-578. [CrossRef]

35. Zhai, S.M. Characterizing computer input with Fitts' law parameters-The information and non-information aspects of pointing. Int. J. Hum.-Comput. Stud. 2004, 61, 791-809. [CrossRef]

36. Peng, C.; Wang, D.; Zhang, Y.; Li, T. Quantifying differences between five fingers in speed-accuracy tradeoff for force control tasks. In Proceedings of the 2017 IEEE World Haptics Conference (WHC), Munich, Germany, 6-9 June 2017; pp. 275-280.

37. Bongers, R.M.; Fernandez, L.; Bootsma, R.J. Linear and logarithmic speed-accuracy trade-offs in reciprocal aiming result from task-specific parameterization of an invariant underlying dynamics. J. Exp. Psychol. Hum. Percept. Perform. 2009, 35, 1443-1457. [CrossRef]

38. Esterman, M.; Noonan, S.K.; Rosenberg, M.; Degutis, J. In the zone or zoning out? Tracking behavioral and neural fluctuations during sustained attention. Cereb. Cortex. 2013, 23, 2712-2723. [CrossRef] [PubMed]

39. Teather, R.J.; Natapov, D.; Jenkin, M. Evaluating haptic feedback in virtual environments using ISO 9241-9. In Proceedings of the 2010 IEEE Virtual Reality Conference (VR), Waltham, MA, USA, 20-24 March 2010; pp. 307-308.

40. Bonneviale, C.; Thon, B. Two-choice reaction time as a function of finger, finger pairing and foreperiod duration. Eur. Bull. Cognit. Psychol. 1990, 10, 251-267.

41. Miller, J. Discrete versus continuous stage models of human information processing: In search of partial output. J. Exp. Psychol. Hum. Percept. Perform. 1982, 8, 273-296. [CrossRef] [PubMed]

42. Schieber, M.H.; Poliakov, A.V. Partial Inactivation of the Primary Motor Cortex Hand Area: Effects on Individuated Finger Movements. J. Neurosci. 1998, 18, 9038-9054. [CrossRef] [PubMed]

43. Schieber, M. Muscular production of individuated finger movements: The roles of extrinsic finger muscles. J. Neurosci. 1995, 15, 284-297. [CrossRef] [PubMed]

44. Szychlinska, M.A.; Dullaert, K.; Beumer, A.; Nsir, H.; Guglielmino, C.; Mazzone, V.; Giunta, S. Ergonomics of Prehensility in Pushing and Pulling Motions: An Anatomical and Biomechanical Overview. J. Funct. Morphol. Kinesiol. 2017, 2, 6. [CrossRef]

45. Meyer, D.E.; Abrams, R.A.; Kornblum, S.; Wright, C.E.; Al, E. Optimality in human motor performance: Ideal control of rapid aimed movements. Psychol. Rev. 1988, 95, 340-370. [CrossRef]

46. Antal, M.; Szabó, L.Z.; László, I. Keystroke Dynamics on Android Platform. Procedia Technol. 2015, 19, 820-826. [CrossRef]

47. Roy, Q.; Guiard, Y.; Bailly, G.; Lecolinet, É.; Rioul, O. Glass + Skin: An Empirical Evaluation of the Added Value of Finger Identification to Basic Single-Touch Interaction on Touch Screens. In Proceedings of the Ifip Tc.13 International Conference on Human-Computer Interaction, Bamberg, Germany, 14-18 September 2015; pp. 55-71.

48. Goguey, A.; Vogel, D.; Chevalier, F.; Pietrzak, T.; Roussel, N.; Casiez, G. Leveraging Finger Identification to Integrate Multi-touch Command Selection and Parameter Manipulation. Int. J. Hum.-Comput. Stud. 2016, 99, 21-36. [CrossRef]

49. Orozco, M.; Asfaw, Y.; Shirmohammadi, S.; Adler, A.; El Saddik, A. Haptic-based biometrics: A feasibility study. In Proceedings of the 2006 14th Symposium on Haptic Interfaces for Virtual Environment and Teleoperator Systems, Arlington, VA, USA, 25-26 March 2006; pp. 265-271. 
50. Ali, M.L.; Monaco, J.V.; Tappert, C.C.; Qiu, M. Keystroke Biometric Systems for User Authentication. J. Signal Process. Syst. 2016, 86, 175-190. [CrossRef]

51. Mayer, N.H. Clinicophysiologic concepts of spasticity and motor dysfunction in adults with an upper motoneuron lesion. Muscle Nerve. 1997, 20, 1-14. [CrossRef]

52. Liu, X.; Zhu, Y.; Huo, H.; Wei, P.; Wang, L.; Sun, A.; Hu, C.; Yin, X.; Lv, Z.; Fan, Y. Design of Virtual Guiding Tasks with Haptic Feedback for Assessing the Wrist Motor Function of Patients with Upper Motor Neuron Lesions. IEEE Trans. Neural Syst. Rehabil. Eng. 2019, 27, 984-994. [CrossRef] [PubMed]

53. Yokoe, M.; Okuno, R.; Hamasaki, T.; Kurachi, Y.; Akazawa, K.; Sakoda, S. Opening velocity, a novel parameter, for finger tapping test in patients with Parkinson's disease. Park. Relat. Disord. 2009, 15, 440-444. [CrossRef] [PubMed]

54. Tavares, A.L.T.; Jefferis, G.S.; Koop, M.; Hill, B.C.; Hastie, T.; Heit, G.; Bronte-Stewart, H.M. Quantitative measurements of alternating finger tapping in Parkinson's disease correlate with UPDRS motor disability and reveal the improvement in fine motor control from medication and deep brain stimulation. Mov. Disord. 2005, 20, 1286-1298. [CrossRef] [PubMed]

(C) 2019 by the authors. Licensee MDPI, Basel, Switzerland. This article is an open access article distributed under the terms and conditions of the Creative Commons Attribution (CC BY) license (http://creativecommons.org/licenses/by/4.0/). 